\title{
Sleep quality among teenagers and young adults with cancer
}

Fortmann. Judith, BSc, MSc; Fisher. Abigail. BSc, PhD Lecturer; Hough. Rachael, MD; Gregory. Alice, M. PhD; Pugh. Gemma. BSc, PhD

\section{Author Affiliations}

Department of Behavioural Science and Health, University College London, 1-19 Torrington Place, London, WC1E 6BT, United Kingdom (Ms Fortmann, Dr Fisher and Dr Pugh)

University College London Hospital's NHS Foundation Trust, 235 Euston Rd, London NW1 2BU, United Kingdom (Dr Hough); Department of Psychology, Goldsmiths, University of London, Lewisham Way, New Cross, London SE14 6NW, United Kingdom (Dr Gregory); and Centre for Sports and Exercise Medicine, William Harvey Research Institute, School of Medicine and Dentistry, Queen Mary University of London (Dr Pugh)

\section{Correspondence}

Dr Gemma Pugh

Email gemma.pugh.14@ucl.ac.uk

Energy Balance and Cancer Research Group, Department of Behavioural Science and Health. University College London, 1-19 Torrington Place, London, WC1E 6BT

\section{Conflicts of Interest}

The authors have no funding or conflicts of interest to disclose

\section{Word Count}

Abstract 241

Main Text 3042

Tables 4

Figures 1

Short Running Title: Sleep quality among teenage and young adult cancer survivors Key Words

Sleep quality; Cancer; Adolescents; Survivorship 


\begin{abstract}
Background: Teenagers and young adults (TYAs) with cancer are known to suffer poor sleep quality and sleep disturbances; understanding the level of burden is essential to improving patient outcomes via supportive care interventions.
\end{abstract}

Study Objectives: to compare sleep quality and the prevalence of sleep disturbances among TYA cancer patients, TYA survivors and general population TYAs with no history of cancer. Methods: TYA patients receiving active cancer treatment $(n=70)$, TYA cancer survivors $(n=151)$ and general population TYAs $(n=324)$ aged between 13 and 24 years completed the Pittsburgh Sleep Quality Index (PSQI). ANCOVAs were used to investigate potential group differences. Age at survey and diagnosis, gender, ethnicity and health status were included as covariates.

Results: $84.29 \%$ of TYA patients, $62.91 \%$ of TYA cancer survivors, and $65.12 \%$ of general population TYAs reported PSQI scores above 5, suggesting clinically significant sleep disorders. TYA patients reported significantly poorer global sleep quality compared to TYA survivors (mean difference $=0.99,95 \% \mathrm{CI}=0.03-1.96, \mathrm{p}=0.044)$ and general population TYAs (mean difference $=1.34,95 \% \mathrm{CI}=0.26-2.41, \mathrm{p}=0.009$ ). TYA patients and survivors reported significantly poorer sleep latency $(\mathrm{p}=0.003$ for TYA patients, $\mathrm{p}=0.035$ for TYA survivors off treatment) and habitual sleep efficiency ( $\mathrm{p} 0.001$ for TYA patients, $\mathrm{p}=0.014$ for TYA survivors) than general population controls.

Conclusions: The significant differences observed suggest young people with cancer, particularly those on treatment, may benefit from specialised sleep interventions.

Implications for Practice: Efforts to ensure health professionals have the knowledge and skills to provide advice about sleep to young people with cancer are needed. 


\section{Introduction}

Seven teenagers or young adults (TYA) are diagnosed with cancer in the United Kingdom (UK) each day. ${ }^{1}$ Advances in cancer therapies mean the overall five-year survival rate among TYAs diagnosed with cancer now exceeds $80 \% .^{2}$ However, cure often comes at a physical and emotional cost with young people suffering treatment- and cancer-related side effects ${ }^{3}$. Poor sleep quality and sleep disturbances have been identified as one of the most common side effects reported by young people during and following cancer treatment. ${ }^{4,5}$ Although the link is not clear, sleep problems in young people with cancer may result from direct effects of the cancer, such as a tumour causing brain damage, or from indirect effects, including treatment side effects, hospitalization, pain, fatigue and anxiety. ${ }^{6}$ If left untreated sleep problems which manifest during treatment may persist into survivorship, potentially resulting in poor quality of life and daily functioning. Specifically within one study of adult survivors of childhood cancer (CCS) $(\mathrm{n}=122$, mean age: 31 years, mean time since diagnosis: 20.5 years) poor sleep efficiency $(<85 \%)$ was significantly associated with lower general, physical and emotional functioning. ${ }^{7}$

Overall sleep quality is comprised of multiple facets including subjective sleep quality, sleep latency, sleep duration, sleep efficiency, sleep disturbances, medication use and daytime dysfunction. ${ }^{8}$ Within the general population poor sleep quality during adolescence and young adulthood has been linked to poorer cognitive functioning, ${ }^{9}$ school performance and learning, ${ }^{10}$ and increased risk of several health problems including obesity and cardiovascular disease. ${ }^{11}$ Adolescents aged 13 to 18 years are recommended 8-10 hours of sleep per night whilst younger adults aged 18- 25 years are recommended 7-9 hours ${ }^{12}$. Data from studies across the globe indicate young people within the general population often fail to meet these recommendations. 
A recent meta-analysis of 41 studies found $53 \%$ of adolescents had insufficent sleep ( $<8$ hours) on school nights with $36 \%$ reporting difficulties falling asleep ${ }^{13}$.

Although there are mixed data and further research is needed to understand the complex associations between sleep and cancer sleep difficulties among cancer patients and survivors are concerning ${ }^{14}$. Among adult cancer survivors, poor sleep quality, circadian rhythm disregulation (caused by sleep disruption) and low levels of sleep duration are thought to contribute to immune dysfunction ${ }^{15}$, neurocogntive impairments ${ }^{16}$, and cancer progression ${ }^{17}$ via inflammatory and oxidative stress pathways ${ }^{17}$. Among children and young people, there is cross-sectional evidence that poor sleep quality is associated with poorer cancer-related quality of life, pain, anxiety and cognitive problems ${ }^{5,18}$.

However, data on the sleep quality of TYA aged cancer patients and survivors is predominantly limited to studies without control groups or studies involving CCS. ${ }^{5,19}$ One study found that among TYA survivors $41 \%$ reported prolonged sleep onset latency ( $<30 \mathrm{~min})$, and 33\% reported both poor sleep efficiency and insufficient sleep duration ( $<8$ hours) in their first year after treatment ${ }^{5}$. Data from the US CCS Cohort indicate CCS $(n=1897)$ have significantly poorer sleep quality more than 30 years after treatment compared to healthy siblings $(n=369)^{19}$. Such data suggest TYA cancer patients and survivors might benefit from supportive care interventions to address poor sleep quality. However, while the majority of TYA cancer patients and survivors receive their cancer diagnosis during adolescence or young adulthood, ${ }^{20}$ CCS will, by definition, have been diagnosed during childhood. Thus, results of studies investigating CCS cannot be directly extrapolated to TYAs due to their unique clinical features. ${ }^{21}$ It is therefore important to first accurately characterize the extent of sleep problems among TYAs with cancer before developing potential interventions. 
The aim of this study was to compare sleep quality between TYA cancer patients (defined as those diagnosed with cancer, currently undergoing treatment), TYA cancer survivors (those diagnosed with cancer who are no longer undergoing active treatment) and general population TYAs (TYAGP) with no history of cancer. Understanding differences between TYA patients, TYA cancer survivors, and TYAGP may strengthen the rationale behind providing TYAs with cancer with specialised sleep interventions to improve sleep quality during and after treatment. Additionally, understanding similarities and differences between groups would help dictate the extent to which interventions developed for TYAGP could be shared, or the extent they need to be tailored and specific to those with cancer.

\section{Methods}

\section{Study design and participants}

In 2015-2016 TYA cancer patients and survivors in the United Kingdom (defined as any young person between the age of 13 and 24 years living with or beyond cancer) and TYAGPs were invited to participate in a cross-sectional survey study investigating their health behaviours, well-being and interest in lifestyle advice. TYA cancer patients and survivors were recruited through CLIC Sargent, a cancer charity in the UK for young people and their families, and outpatient clinics at University College Hospital London. TYAs were eligible to participate regardless of their cancer type, age at diagnosis or stage in the cancer care continuum. Terminally ill TYA cancer patients and those who received palliative care were excluded from the study. Detailed information regarding the recruitment procedure of TYA patients and survivors can be found in previous publications ${ }^{22,23}$. TYAGPs were recruited online vie social media and research participation platforms, and through schools, community and youth groups in the area of greater London. TYAGPs were eligible to take part if they were aged between 13 and 24 years, were living in the UK during the time of data collection and understood spoken 
and written English. Participants could complete the survey either in paper format or via an online link. After finishing the questionnaire, participants had the opportunity to be included in a prize draw to win vouchers worth $£ 15-£ 50$. Ethical approval was obtained from UCL Research Ethics Committee (project number: 6206/001) and London Hampstead NHS Research Ethics Committee (reference: 15/LO/0764).

\section{Measures}

\section{Demographic Information and Health Characteristics}

Self-report data on age, gender and ethnicity were collected alongside data on additional health problems from TYA patients, survivors and GPTYAs. Data on stage at cancer diagnosis, cancer type, cancer stage, treatment type, treatment stage and time since treatment from TYA patients and survivors. Since chemotherapy and radiotherapy are known to have a negative impact on sleep ${ }^{24}$ TYA patients and survivors were classified as having received/receiving chemotherapy and/or radiotherapy or not having received/receiving either of the two.

Sleep

Sleep quality over the past month was measured using the Pittsburgh Sleep Quality Index (PSQI). The 19-question scale has seven dimensions: subjective sleep quality, sleep latency (the length of time it takes to fall asleep), sleep duration, habitual sleep efficiency (the ratio of the total time spent asleep per night compared to the total amount of time spent in bed), sleep disturbances, sleep medication use and daytime dysfunction. The individual dimensions can be categorised into scores ranging from zero to three. They can be evaluated separately, or combined to give a global score ranging from zero to 21 , with greater scores indicating poorer sleep quality. The PSQI is validated for use among cancer patients (Cronbach's $\alpha=0.81$ ) and has shown good reliability $(r=0.85) .{ }^{8}$ A cut-off score of five identifies cases with sleep problems, which may warrant clinical attention. ${ }^{25}$ 


\section{Statistical analysis}

Participants who did not complete any items contained within the PSQI were excluded from all analyses. Where participants completed the PSQI partially, missing data were imputed using expectation maximization algorithm. Expectation maximization algorithm was also used to impute missing data for continuous demographic variables and health characteristics. Categorical demographics and health characteristics were not imputed to reduce bias. Instead pairwise deletion was applied to minimise loss of data. ${ }^{26}$

Descriptive statistics were calculated for all variables. $\chi^{2}$ tests, two-sample t-tests and ANOVAs were used to test for differences in demographics and health characteristics between TYACS and TYAGP. Following the advice of the TYA clinical team TYACS were stratified into TYA cancer patientsand TYA survivors. Although both groups are widely referred to as TYA cancer survivors, they differ in their medical and supportive care needs. ${ }^{27}$ TYA cancer patients might experience hospitalization and treatment-related side effects, whilst TYA cancer survivors can be faced with challenges involving reintegration with their peers and regaining a sense of normalcy to their lives, sleep quality may therefore be different between the two groups.

Prior to main analyses sleep latency, duration, efficiency and disturbances, and medication use were $\log$ and square root transformed to reduce the non-normal distribution of data. Twosample t-tests and ANOVAs were used for unadjusted comparisons of individual sleep components and global sleep quality, then ANCOVAs adjusting for covariates were run. In adjusted comparisons involving TYAGP demographics and (additional) health problems were entered as covariates. The presence of health problems was coded as No health problem present, One health problem present or More than two health problems present. 
Comparisons between TYA patients and cancer survivors were additionally adjusted for age at diagnosis. Other cancer-specific health characteristics such as cancer type were not controlled for, as no significant associations between these and any of the key variables were found in preliminary analyses.

All data were analysed using IBM SPSS Version 24.0. The significance level was set at $\alpha=$ 0.05 for all analyses. $95 \%$ confidence intervals $(95 \% \mathrm{CI})$, and mean differences as effect size estimates, were reported. Based on studies investigating sleep quality among CCS compared to the general population, medium effect sizes were expected. ${ }^{17}$ Power calculations suggested samples of minimum 64 participants per group (ANCOVA, medium effect size $(\mathrm{f}=0.25)$, power of $80 \%, \alpha=0.05)$.

\section{Results}

\section{Response rate}

In total, 295 young people with cancer (70 TYA cancer patients receiving active treatment; 151 TYA cancer survivors who had completed treatment) and 370 TYAGP completed the health and lifestyle questionnaire. Sixty young people with cancer and 46 TYAGP did not complete any items contained within the PSQI, and were excluded from analyses. Nine young people with cancer did not indicate their treatment status.

\section{Sample and health characteristics}

Table 1 and 2 provide an overview of the demographic information of the study population, and their health characteristics, respectively. The average age at survey for TYA patients was 19.43 years $(\mathrm{SD}=3.12), 20.06$ years $(\mathrm{SD}=2.82)$ for TYA cancer survivors, and 17.10 years $(\mathrm{SD}=3.10)$ for TYAGP. TYA patients and survivors were significantly older than TYAGP 
(both $\mathrm{p}<0.001)$. The majority of participants in all groups were female $(58.57 \%$ TYA cancer patients, $60.93 \%$ TYA cancer survivors, and $77.77 \%$ for TYAGP) but the percentage of females was significantly higher among TYAGP $(\mathrm{p}=0.003)$ when compared to TYA patients and survivors. The majority of young people with cancer were White $(85.07 \%$ TYA patients, $86.67 \%$ TYA survivors); the TYAGP were more ethnically diverse (White $=51.23 \%$, Black $=26.85 \%$, Asian=8.33\% and Mixed $=11.11 \%$ ) (both $\mathrm{p}<0.001)$. The majority of young people with cancer reported extreme fatigue $(35.71 \%$ TYA cancer patients; $27.15 \%$ TYA survivors) and mental health issues (14.29\% TYA patients; $18.54 \%$ TYA survivors) as additional health problems. The most common health problems among TYAGP were mental health problems $(14.89 \%)$ and asthma $(12.65 \%)$. The majority of TYA cancer survivors (45.70\%) had finished their treatment between one and five years prior to survey.

\section{Sleep quality}

Table 3 lists the mean scores for the individual sleep components and global sleep quality, and Table 4 provides an overview of unadjusted group comparisons. Figure 1 displays comparisons adjusted for covariates. Overall, $84.29 \%$ of TYA patients, $62.91 \%$ of TYA cancer survivors and $64.81 \%$ of TYAGP reported global PSQI scores above five, suggesting potential sleep problems. The average global PSQI score was $8.64(\mathrm{SD}=3.59)$ for TYA cancer patients, 7.20 for TYA cancer survivors ( $\mathrm{SD}=3.49)$ and 6.97 for TYAGP (SD=3.16). In comparisons adjusted for covariates TYA cancer patients showed significantly poorer global sleep quality than TYA cancer survivors (mean difference $=0.99,95 \% \mathrm{CI}=0.03-1.96, \mathrm{p}=0.044$ ) and TYAGP (mean difference $=1.34,95 \% \mathrm{CI}=0.26-2.41, \mathrm{p}=0.009)$. No difference was found between TYA cancer survivors and TYAGP. 
In relation to the individual sleep components, in adjusted comparisons TYA cancer patients reported significantly worse subjective sleep quality than TYA cancer survivors (mean difference $=0.19,95 \% \mathrm{CI}=0.02-0.37, \mathrm{p}=0.033)$. The average sleep latency for TYA cancer patients was 39.76 minutes ( $\mathrm{SD}=35.15$ minutes), and 34.55 minutes ( $\mathrm{SD}=35.42$ minutes) for TYA cancer survivors. In adjusted comparisons, both TYA patients (mean difference $=0.27$, $95 \% \mathrm{CI}=0.08-0.47 \mathrm{p}=.003$ ) and TYA cancer survivors (mean difference $=0.17,95 \% \mathrm{CI}=0.01-$ $0.33, \mathrm{p}=.035$ ) reported significantly greater sleep latency compared to TYAGP who took on average 29.88 minutes ( $\mathrm{SD}=42.68$ minutes) to fall asleep. TYAGPs' sleep length was on average 7.11 hours ( $\mathrm{SD}=1.52$ hours) which was significantly shorter compared to TYA cancer patients (mean difference $=-0.30,95 \% \mathrm{CI}=-0.57--0.04, \mathrm{p}=.026$ ) and survivors who reported average sleep durations of 7.83 hours ( $\mathrm{SD}=1.65$ hours) and 7.92 hours ( $\mathrm{SD}=1.32$ hours). TYA patients, TYA survivors and TYAGP reported average sleep efficiency was $80.81 \%$ $(\mathrm{SD}=14.84 \%), 85.42 \%(\mathrm{SD}=11.21 \%)$ and $87.30 \%(\mathrm{SD}=13.41 \%)$, with TYAGP showing significantly greater sleep efficiency than TYA cancer patients (mean difference $=0.33,95 \%$ $\mathrm{CI}=0.14-0.52, \mathrm{p}<0.001)$ and survivors (mean difference $=0.18,95 \% \mathrm{CI}=0.03-0.33, \mathrm{p}=.014$ ). TYA cancer patients reporting significantly more sleep disturbances than survivors (mean difference $=0.11,95 \% \mathrm{CI}=0.02-0.19, \mathrm{p}=.012$ ) and TYAGP (mean difference $=0.17,95 \%$ $\mathrm{CI}=0.08-0.26, \mathrm{p}<0.001)$. No group differences in sleep medication or daytime dysfunction were found.

\section{Discussion}

Differences in sleep quality were investigated between TYA cancer patients, TYA cancer survivors and TYAGP. High prevalence of sleep problems was observed across all groups. TYA cancer patients reported significantly worse global sleep quality and more sleep disturbances than TYA cancer survivors and TYAGP. Both TYA cancer patients and survivors 
reported significantly longer sleep latency and shorter sleep efficiency than TYAGP. Sleep duration was shortest among TYAGP. No differences were found in medication use and daytime dysfunction.

The high prevalence of potential sleep problems observed among young people with cancer and the general population reflect existing studies which indicate sleep quality among adolescents and young adults is poor ${ }^{13}$. Among general population adolescents poor sleep quality is attributed to delayed sleep onset, irregular sleep patterns, and poor sleep hygiene behaviours such as use of electronic devices whilst in bed. ${ }^{9,28}$ Poor sleep quality observed among TYA cancer patients and survivors within this study reflect existing data from paediatric cancer patients and adult aged $\mathrm{CCS}^{4-6,18,29,30}$. The difference in global sleep quality was larger between TYA cancer patients and TYAGP (medium effect size=1.34) compared to TYA cancer patients and TYA cancer survivors (medium effect size=0.99). This is likely due to greater cancer-specific differences between TYA cancer patients and TYAs with no history of cancer. The difference in global sleep quality observed between TYA cancer patients and survivors may be explained by certain factors unique to TYACS on treatment, including medicationrelated sickness, potentially stronger impacts of cancer-related fatigue and pain, and increased sleep disturbances such as awakening during the night due to hospitalisation. Anxiety resulting from the cancer diagnosis may also play a role. ${ }^{31}$ Poor sleep quality among young people with cancer during and after treatment is concerning as it is likely to impact the young persons' resilience and coping ability to deal with common side effects of treatment such as depression, anxiety, pain and functional impairment. ${ }^{6,32}$ Interventions that improve quality of sleep among TYA cancer patients and survivors are likely to improve emotional and psychosocial outcomes within this group. 
Sleep duration was found to be significantly shorter among TYAGP compared to young people with cancer. The mean age of TYA cancer patients and survivors who participated in this study was within the young adult age range; therefore in this study young people with cancer were classified as meeting the recommended sleep duration of seven to nine hours. ${ }^{12}$ In contrast the mean age of TYAGP was within the teenage age group; therefore, in this study TYAGP were classified as not meeting the recommendation of eight to ten hours. There may be a need for interventions to prolong sleep duration among TYAGP. However, previous studies suggest that shorter sleep may result in better sleep quality. Furthermore, suffering from cancer may result in increased sleepiness, ${ }^{33}$ which may explain the difference found between TYACS and TYAGP.

Although TYA cancer survivors reported significantly prolonged sleep latency and poorer efficiency, no differences were found in global sleep quality, which contradicts previous studies that found poorer sleep quality among CCS compared to the general population. ${ }^{19}$ However, considering that adolescents and young adults are a unique age group, comparisons with other age groups should be made with caution. Furthermore, given that the majority of TYA cancer survivors included in this study had finished all active treatment between one and five years prior to survey they may have sufficiently recovered from their cancer and its associated effects by the time of study. An alternative explanation may be a response shift potentially experienced by TYA cancer survivors, i.e. a 'change of internal standards and values. ${ }^{34}$ Shortly after having finished treatment young people with cancer may have lowered standards of health, and the quality of sleep reported may not truly reflect their actual sleep quality. 
The findings of this study suggest young people with cancer, TYA cancer patients in particular, may benefit from a sleep intervention. However, there is a lack of effective sleep interventions among young people with cancer. While physical activity and massage therapies have been suggested as potential interventions to improve sleep quality evidence regarding their effectiveness is restricted to pilot studies or trials without control groups. ${ }^{35,36}$ Among general population adolescents cognitive behavioural therapy and mindfulness group interventions have demonstrated positive effects on behaviour and sleep quality. ${ }^{37}$ There is a need for randomised controlled trails investigating whether sleep interventions, which have demonstrated positive effect among the general population could be have warranted among young people with cancer. However, it is essential such interventions are tailored to the specific needs of TYA cancer patients and survivors, and account for cancer-related factors such as pain, nausea and hospitalisation.

Considering the detrimental effects poor sleep quality may have, health professionals should emphasise the importance of good sleep quality and promote the uptake of interventions to improve sleep outcomes among young people with cancer. However, data from Project REACH ( $n=122$ adult survivors of childhood cancer, mean age: 31 years) indicate two out of three patients who suffer self-report insomnia do not discuss the issue with their medical team. ${ }^{38}$ This may reflect reluctance among patients to discuss sleep problems and lack of knowledge regarding the assessment and provision of advice about sleep problems by health care professionals. Future research is needed to investigate these potential issues.

Although this is the first study to compare sleep quality among TYA cancer patients, TYA cancer survivors and TYAGP, several limitations need to be considered when interpreting the findings. First, sleep quality was measured using a self-report questionnaire, therefore 
introducing potential reporting bias including over- and underestimation of sleep duration and latency. Second, young people with cancer and general population controls differed significantly in demographics and sample size, thereby reducing comparability of the groups. Third, the study used a UK sample of TYAs aged between 13 and 24 years. Findings are therefore limited in generalizability to countries that apply different age ranges to define TYA survivors, such as the United States.

Future research should aim to compare sleep quality among larger groups that are better matched in sample size and demographics, and include objective measures such as polysomnography. ${ }^{39}$ Such studies should also include large numbers of participants per cancer and treatment type to investigate whether poor sleep quality is associated with any demographic or cancer-related variables, to identify those who might benefit most from an intervention.

In conclusion, the present study has identified potential impairments in sleep quality among TYA cancer patients compared to TYA cancer survivors and TYAGP. In addition, the high prevalence of possible sleep problems indicates poor sleep quality is prevalent among all groups. Due to a current lack of effective interventions for adolescents and young adults with cancer research developing sleep interventions tailored to this population, particularly for those undergoing treatment, is warranted. Such interventions may improve the general, physical and emotional functioning of TYA cancer patients and survivors.

\section{Acknowledgements}

\section{Conflicts of Interests}

The authors declare that there are no competing interests. 


\section{Institutional Review}

This study was approved by The University College London Ethics Committee 6206/001 and London Hampstead NHS Research Ethics Committee 15/LO/0764. Informed consent was obtained from all individual participants included in the study. By completing and returning the health and lifestyle questionnaire young people were consenting to their data being used for the purposes outlined within this study.

\section{Submission Declaration}

All phases of the study were supported by an IMPACT Studentship co-funded by CLIC Sargent and University College London awarded to GP. Cancer Research UK funded the survey printing. GP is supported by a Pan-London Research Fellowship funded by RM Partners, UCLH Cancer Collaborative and South East London Accountable Cancer Network (SEL CAN) on behalf of the National Cancer Vanguard. This work has not been published previously. 


\section{References}

1. O'Hara C, Moran A, Whelan JS, et al. Trends in survival for teenagers and young adults with cancer in the UK 1992-2006. European Journal of Cancer. 9// 2015;51(14):2039-2048.

2. Stark D, Bowen D, Dunwoodie E, et al. Survival patterns in teenagers and young adults with cancer in the United Kingdom: Comparisons with younger and older age groups. European journal of cancer. 2015;51(17):2643-2654.

3. Brinkman TM, Li C, Vannatta K, et al. Behavioral, Social, and Emotional Symptom Comorbidities and Profiles in Adolescent Survivors of Childhood Cancer: A Report From the Childhood Cancer Survivor Study. Journal of Clinical Oncology. July 18, 20162016.

4. Mandrell BN, Moore C, Crabtree VM. Assessing Common Sleep Disturbances in Survivors of Childhood Cancer. The Journal for Nurse Practitioners. 2018/04/01/ 2018;14(4):245-250.

5. Daniel LC, Aggarwal R, Schwartz LA. Sleep in Adolescents and Young Adults in the Year After Cancer Treatment. J Adolesc Young Adult Oncol. Dec 2017;6(4):560-567.

6. Walter L, Nixon G, Davey M, Downie P, Horne RSC. Sleep and fatigue in pediatric oncology: A review of the literature. Sleep medicine reviews. 2015;24:71-82.

7. Zhou ES, Recklitis CJ. Insomnia in adult survivors of childhood cancer: a report from project REACH. Supportive Care in Cancer. 2014/11/01 2014;22(11):3061-3069.

8. Beck SL, Schwartz AL, Towsley G, Dudley W, Barsevick A. Psychometric evaluation of the Pittsburgh Sleep Quality Index in cancer patients. J Pain Symptom Manage. Feb 2004;27(2):140-148.

9. Owens J. Insufficient Sleep in Adolescents and Young Adults: An Update on Causes and Consequences. Pediatrics. 2014.

10. Dewald JF, Meijer AM, Oort FJ, Kerkhof GA, Bogels SM. The influence of sleep quality, sleep duration and sleepiness on school performance in children and adolescents: A meta-analytic review. Sleep Med Rev. Jun 2010;14(3):179-189.

11. Shochat $T$, Cohen-Zion $M$, Tzischinsky O. Functional consequences of inadequate sleep in adolescents: a systematic review. Sleep Med Rev. Feb 2014;18(1):75-87.

12. Paruthi S, Brooks LJ, D'Ambrosio C, et al. Recommended Amount of Sleep for Pediatric Populations: A Consensus Statement of the American Academy of Sleep Medicine. Journal of Clinical Sleep Medicine : JCSM : Official Publication of the American Academy of Sleep Medicine. 06/15

04//received

04//accepted 2016;12(6):785-786.

13. Gradisar M, Gardner G, Dohnt H. Recent worldwide sleep patterns and problems during adolescence: A review and meta-analysis of age, region, and sleep. Sleep Medicine. 2011/02/01/2011;12(2):110-118.

14. Erren TC, Morfeld P, Foster RG, et al. Sleep and cancer: synthesis of experimental data and meta-analyses of cancer incidence among some 1,500,000 study individuals in 13 countries. Chronobiology international. 2016; 33(4), 325-350.

15. Sephton S, Spiegel D. Circadian disruption in cancer: a neuroendocrine-immune pathway from stress to disease? Brain Behav Immun. Oct 2003;17(5):321-328. 
16. Cheung YT, Lim SR, Ho HK, Chan A. Cytokines as mediators of chemotherapyassociated cognitive changes: current evidence, limitations and directions for future research. PLoS One. 2013;8(12):e81234.

17. Nerozzi D, Santoni A, Bersani G, et al. Reduced natural killer cell activity in major depression: neuroendocrine implications. Psychoneuroendocrinology. 1989;14(4):295-301.

18. Erickson JM, Beck SL, Christian BR, et al. Fatigue, sleep-wake disturbances, and quality of life in adolescents receiving chemotherapy. J Pediatr Hematol Oncol. Jan 2011;33(1):e17-25.

19. Mulrooney DA, Ness KK, Neglia JP, et al. Fatigue and Sleep Disturbance in Adult Survivors of Childhood Cancer: A Report from the Childhood Cancer Survivor Study (CCSS). Sleep. 02//received 07//accepted 2008;31(2):271-281.

20. Stark D, Bowen D, Dunwoodie E, et al. Survival patterns in teenagers and young adults with cancer in the United Kingdom: Comparisons with younger and older age groups. European Journal of Cancer. 2015;5(17), 2643-2654.

21. Zebrack BJ. Psychological, social, and behavioral issues for young adults with cancer. Cancer. May 15 2011;117(10 Suppl):2289-2294.

22. Pugh G, Hough RE, Gravestock HL, Jackson SE, Fisher A. The Health Behavior Information Needs and Preferences of Teenage and Young Adult Cancer Survivors. Journal of Adolescent and Young Adult Oncology. 2017;6(2):318 - 326

23. Pugh G, Hough R, Gravestock H, Haddrell JB, Beeken RJ, Fisher A. The Lifestyle Information and Intervention Preferences of Teenage and Young Adult Cancer Survivors: A Qualitative Study. Cancer Nurs. Jun 152017.

24. Walter LM, Nixon GM, Davey MJ, Downie PA, Horne RS. Sleep and fatigue in pediatric oncology: A review of the literature. Sleep Med Rev. Dec 2015;24:71-82.

25. Buysse DJ, Reynolds CF, 3rd, Monk TH, Berman SR, Kupfer DJ. The Pittsburgh Sleep Quality Index: a new instrument for psychiatric practice and research. Psychiatry Res. May 1989;28(2):193-213.

26. Graham JW. Missing data analysis: making it work in the real world. Annu Rev Psychol. 2009;60:549-576.

27. Barr RD, Ferrari A, Ries L, Whelan J, Bleyer W. Cancer in adolescents and young adults: A narrative review of the current status and a view of the future. JAMA Pediatrics. 2016;170(5):495-501.

28. Bruce ES, Lunt L, McDonagh JE. Sleep in adolescents and young adults. Clinical Medicine. 2017;17(5):424-428.

29. Brimeyer C, Adams L, Zhu L, et al. Sleep complaints in survivors of pediatric brain tumors. Supportive Care in Cancer. 2016;24(1):23-31.

30. Desaulniers G, Riley L, Vangile K, et al. Self-Reported Sleep Problems in Adolescent Survivors of Childhood Cancer. Clinical journal of oncology nursing. 2015;19(1):8188.

31. Cella D, Lai JS, Chang $\mathrm{CH}$, et al. Fatigue in cancer patients compared with fatigue in the general United States population. Cancer. 2002;94(2):528-38.

32. Daniel L, Kazak A, Li Y, et al. Relationship between sleep problems and psychological outcomes in adolescent and young adult cancer survivors and controls. Supportive Care in Cancer. 2016;24(2):539-546. 
33. Morin CM, Bootzin RR, Buysse DJ, Edinger JD, Espie CA, Lichstein KL. Psychological And Behavioral Treatment Of Insomnia: Update Of The Recent Evidence (19982004). Sleep. 2006;29(11):1398-1414.

34. Sprangers MA, Schwartz CE. Integrating response shift into health-related quality of life research: a theoretical model. Soc Sci Med. Jun 1999;48(11):1507-1515.

35. Orsey AD, Wakefield DB, Cloutier MM. Physical activity (PA) and sleep among children and adolescents with cancer. Pediatric Blood \& Cancer. 2013;60(11):19081913.

36. Jacobs S, Mowbray C, Cates LM, et al. Pilot Study of Massage to Improve Sleep and Fatigue in Hospitalized Adolescents With Cancer. Pediatr Blood Cancer. May 2016;63(5):880-886.

37. Blake MJ, Snoep L, Raniti M, et al. A cognitive-behavioral and mindfulness-based group sleep intervention improves behavior problems in at-risk adolescents by improving perceived sleep quality. Behav Res Ther. Dec 2017;99:147-156.

38. Zhou ES, Recklitis CJ. Insomnia in adult survivors of childhood cancer: a report from project REACH. Support Care Cancer. Nov 2014;22(11):3061-3069.

39. Sadeh A. The role and validity of actigraphy in sleep medicine: an update. Sleep Med Rev. Aug 2011;15(4):259-267. 
Table 1. Participant demographics

\begin{tabular}{|c|l|l|l|}
\hline & $\begin{array}{l}\text { TYA cancer } \\
\text { patients }(\mathrm{n}=70)\end{array}$ & $\begin{array}{l}\text { TYA cancer } \\
\text { survivors }(\mathrm{n}=151)\end{array}$ & TYAGP (n=324) \\
\hline Age at survey, mean (SD) & $19.43(3.12)$ & $20.06(2.82)$ & $17.10(3.10)$ \\
\hline Gender, \% (n) & & & \\
\hline Female & $58.57(41)$ & $60.93(92)$ & $77.77(252)$ \\
\hline Male & $41.43(29)$ & $39.07(59)$ & $21.92(71)$ \\
\hline Missing & $0(0)$ & $0(0)$ & $0.31(1)$ \\
\hline Ethnicity, \% (n) & & & \\
\hline White & $78.57(55)$ & $82.78(125)$ & $51.23(166)$ \\
\hline Black & $5.71(4)$ & $10.59(16)$ & $26.85(87)$ \\
\hline Asian & $8.57(6)$ & $3.97(6)$ & $8.33(27)$ \\
\hline Mixed & $7.14(5)$ & $2.65(4)$ & $11.11(36)$ \\
\hline Missing & $0(0)$ & $0(0)$ & $2.47(8)$ \\
\hline
\end{tabular}

Abbreviations: TYA, Teenage and Young Adult 
Table 2

Participant health characteristics

\begin{tabular}{|c|c|c|c|}
\hline & $\begin{array}{l}\text { TYA cancer } \\
\text { patients }(n=70)\end{array}$ & $\begin{array}{l}\text { TYA cancer } \\
\text { survivors }(\mathrm{n}=151)\end{array}$ & TYAGP $(n=324)$ \\
\hline No health problem present, $\%(\mathrm{n})$ & $44.3(31)$ & $49.7(76)$ & $58.0(188)$ \\
\hline One health problem present, $\%$ (n) & $37.1(26)$ & $32.5(49)$ & $31.2(101)$ \\
\hline$\geq 2$ health problems present, $\%(\mathrm{n})$ & $18.6(13)$ & $17.9(27)$ & $7.7(25)$ \\
\hline Osteoporosis & $1.4(1)$ & $4.0(6)$ & $0.3(1)$ \\
\hline Diabetes & $7.1(5)$ & $2.6(4)$ & $0.9(3)$ \\
\hline Asthma & $10.0(7)$ & $11.3(17)$ & $12.7(41)$ \\
\hline Irregular heart rhythm & $7.1(5)$ & $5.3(8)$ & $1.9(6)$ \\
\hline Extreme fatigue & $35.7(25)$ & $27.2(41)$ & $6.5(21)$ \\
\hline Mental health problems & $14.3(10)$ & $18.5(28)$ & $14.8(48)$ \\
\hline Lung disease & $0(0)$ & $0.7(1)$ & $0.3(1)$ \\
\hline Arthritis & $1.4(1)$ & $2.6(4)$ & $06(2)$ \\
\hline Any other heart trouble & $1.4(1)$ & $2.0(3)$ & $1.2(4)$ \\
\hline (Another) cancer & $2.9(2)$ & $1.3(2)$ & $0.6(2)$ \\
\hline Sensory impairment & $1.4(1)$ & $3.3(5)$ & $0.9(3)$ \\
\hline Other & & & $7.7(25)$ \\
\hline Age at diagnosis, mean (SD) & $17.46(3.96)$ & $15.78(4.46)$ & \\
\hline \multicolumn{4}{|l|}{ Treatment type, \% (n) } \\
\hline CT or RT & $92.86(65)$ & $94.70(143)$ & \\
\hline No CT or RT & $7.14(5)$ & $5.30(8)$ & \\
\hline \multicolumn{4}{|l|}{ Cancer type, \% (n) } \\
\hline Leukaemia & $34.29(24)$ & $27.2(41)$ & \\
\hline Lymphoma & $25.7(18)$ & $36.42(55)$ & \\
\hline Carcinoma & $0(0)$ & $4.0(6)$ & \\
\hline Germ cell tumour & $0(0)$ & $4.0(6)$ & \\
\hline CNS tumour & $8.6(8)$ & $5.3(8)$ & \\
\hline Melanoma & $1.4(1)$ & $0(0)$ & \\
\hline Bone tumour & $8.6(8)$ & $10.6(16)$ & \\
\hline Soft tissue tumour & $15.7(11)$ & $3.3(5)$ & \\
\hline Other & $5.7(4)$ & $8.6(13)$ & \\
\hline Missing & $0(0)$ & $0.7(1)$ & \\
\hline \multicolumn{4}{|l|}{ Cancer stage, \% (n) } \\
\hline 0 & $0(0)$ & $0.7(1)$ & \\
\hline $\mathrm{I}$ & $10.0(7)$ & $6.6(10)$ & \\
\hline II & $11.4(8)$ & $19.2(29)$ & \\
\hline III & $11.4(8)$ & $15.2(23)$ & \\
\hline IV & $12.9(9)$ & $11.9(18)$ & \\
\hline Don’t know & $51.43(36)$ & $41.72(63)$ & \\
\hline Don't want to answer & $0(0)$ & $2.0(3)$ & \\
\hline Missing & $2.9(2)$ & $2.6(4)$ & \\
\hline
\end{tabular}

Abbreviations: CT, Chemotherapy; RT, Radiotherapy 
Table 3. Mean scores of sleep quality of the study population

\begin{tabular}{|l|l|l|l|}
\hline & $\begin{array}{l}\text { TYA cancer } \\
\text { patients }(\mathrm{n}=70)\end{array}$ & $\begin{array}{l}\text { TYA cancer } \\
\text { survivors }(\mathrm{n}=151)\end{array}$ & TYAGP $(\mathrm{n}=324)$ \\
\hline Subjective sleep quality, mean (SD) & $2.27(0.63)$ & $2.03(0.63)$ & $2.06(0.59)$ \\
\hline Sleep latency, mean (SD) & $1.81(0.97)$ & $1.65(1.23)$ & $1.27(1.01)$ \\
\hline Sleep duration, mean (SD) & $0.61(0.92)$ & $0.44(0.78)$ & $0.84(0.99)$ \\
\hline Sleep efficiency, mean (SD) & $0.87(1.05)$ & $0.63(0.86)$ & $0.38(0.81)$ \\
\hline Sleep disturbances, mean (SD) & $1.50(1.15)$ & $1.17(0.83)$ & $1.03(0.48)$ \\
\hline Medication use, mean (SD) & $0.36(0.92)$ & $0.30(0.81)$ & $0.27(0.68)$ \\
\hline Daytime dysfunction, mean (SD) & $1.21(0.96)$ & $0.99(0.90)$ & $1.13(0.92)$ \\
\hline Global score, mean (SD) & $8.64(3.59)$ & $7.20(3.49)$ & $6.97(3.16)$ \\
\hline
\end{tabular}

${ }^{\mathrm{a}}$ For each measure a higher score indicates poorer sleep quality 
Table 4. Unadjusted comparisons of sleep quality mean scores

\begin{tabular}{|c|c|c|c|}
\hline & $\begin{array}{l}\text { TYA cancer patients vs } \\
\text { TYA cancer survivors }\end{array}$ & $\begin{array}{l}\text { TYA cancer patients } \\
\text { vs. TYAGP }\end{array}$ & $\begin{array}{l}\text { TYA cancer survivors } \\
\text { vs. TYAGP }\end{array}$ \\
\hline \multicolumn{4}{|c|}{ Subjective sleep quality } \\
\hline Mean difference & $0.24^{*}$ & $0.20 *$ & -0.04 \\
\hline $95 \% \mathrm{CI}$ & $0.03-0.45$ & $0.01-0.40$ & $-0.18-0.11$ \\
\hline \multicolumn{4}{|l|}{ Sleep latency } \\
\hline Mean difference & 0.09 & $0.30 * *$ & $0.19 *$ \\
\hline $95 \% \mathrm{CI}$ & $-0.04-0.22$ & $0.14-0.45$ & $0.07-0.31$ \\
\hline \multicolumn{4}{|l|}{ Sleep duration } \\
\hline Mean difference & 0.11 & $-0.30 *$ & $-0.23 * *$ \\
\hline $95 \% \mathrm{CI}$ & $-0.07-0.28$ & $-0.57--0.04$ & $-0.34--0.13$ \\
\hline \multicolumn{4}{|l|}{ Sleep efficiency } \\
\hline Mean difference & 0.11 & $0.37 * *$ & $0.23 * *$ \\
\hline $95 \% \mathrm{CI}$ & $-0.02-0.25$ & $0.22-0.52$ & $0.11-0.34$ \\
\hline \multicolumn{4}{|c|}{ Sleep disturbances } \\
\hline Mean difference & $0.14 *$ & $0.18 * *$ & 0.05 \\
\hline $95 \% \mathrm{CI}$ & $0.04-0.23$ & $0.10-0.27$ & $-0.02-0.11$ \\
\hline \multicolumn{4}{|l|}{ Medication use } \\
\hline Mean difference & 0.02 & 0.01 & -0.01 \\
\hline $95 \% \mathrm{CI}$ & $-0.16-0.19$ & $-0.14-0.17$ & $-0.12-0.11$ \\
\hline \multicolumn{4}{|c|}{ Daytime dysfunction } \\
\hline Mean difference & 0.22 & 0.08 & -0.14 \\
\hline $95 \% \mathrm{CI}$ & $-0.01-0.54$ & $-0.21-0.37$ & $-0.36-0.08$ \\
\hline \multicolumn{4}{|l|}{ Global score } \\
\hline Mean difference & $1.43 *$ & $1.67 * *$ & 0.23 \\
\hline $95 \% \mathrm{CI}$ & $0.28-2.58$ & $0.62-2.72$ & $-0.55-1.02$ \\
\hline
\end{tabular}

$* \mathrm{p}<.05 ; * * \mathrm{p}<.001$ 
Figure 1

Adjusted comparisons of PSQI individual component mean scores

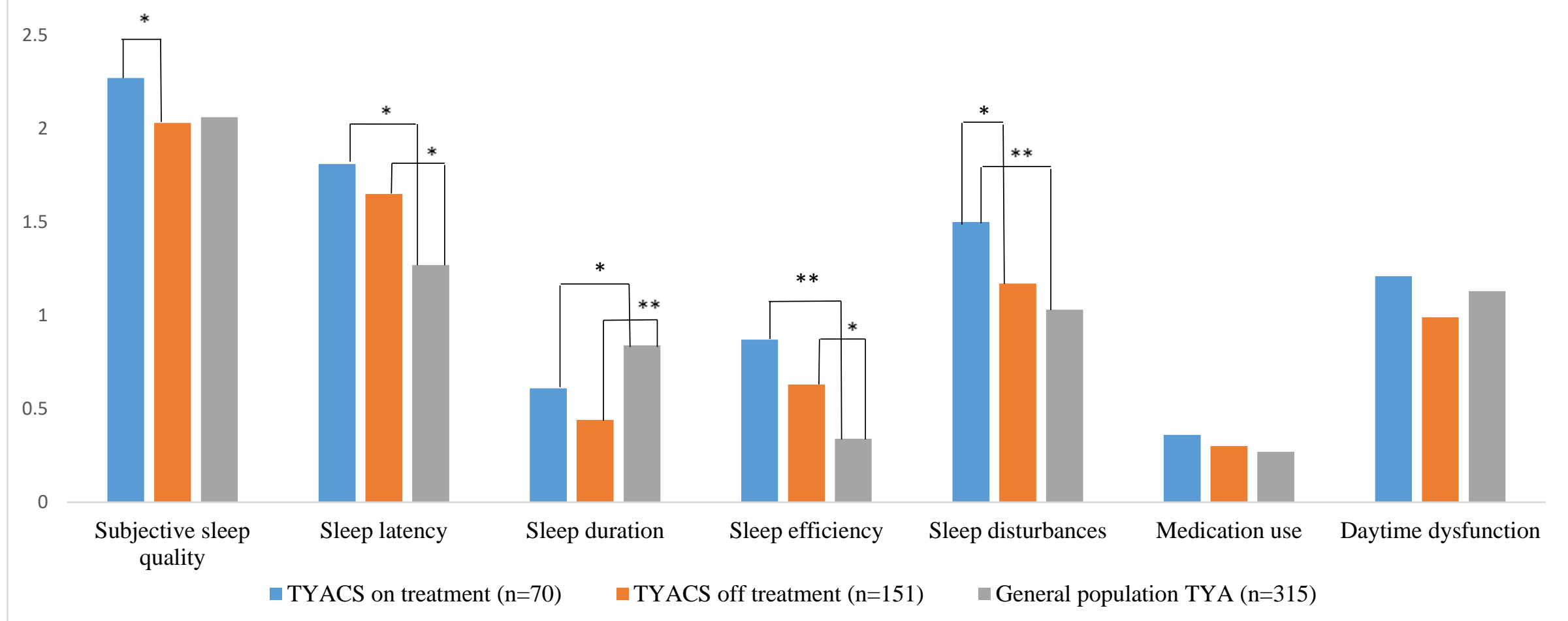

${ }^{\dagger}$ For each measure higher scores indicate poorer sleep quality

$* \mathrm{p}<0.05$

$* * p<0.001$ 